\title{
Why the Coffee Powder is the Best Topical Wound Dressing?
}

\author{
Hendro Sudjono Yuwono
}

\section{ABSTRACT}

The coffee powder has inherent capabilities as an antioxidant, antiinflammatory, and antimicrobial. It is a topical wound dressing for acute and chronic wounds, encouraging results different from the wound dressing known today. It is named the new paradigm of wound management. The study of coffee powder since 2003 as a topical wound dressing has created an understanding condemn to disturb the cells in the wound bed. A thin layer of coffee powder should stay in place to ensure safety, covering superficial wound cells' growth. The remained thin layer of coffee powder has created minimum detached wound cells. Wound healing provides effectiveness for acute and chronic wounds resulted in low cost, easy to get, acceptable scar, non-traumatic, pleasant scent, and not scary to the patients. The utilization of antioxidant, anti-inflammatory, and antibacterial capacities anticipates the injury of the new growth of epithelial cells at the wound bed. It makes better cell proliferation, proper scar formation and safe naturally. Its simplicity in wound management procedures helps improve public health efforts. Hence, it suggested that the coffee powder has the capabilities of the best topical wound dressing.

Keywords: Antibacterial, antioxidant, coffee powder, new paradigm of wound management, wound healing.

\section{INTRODUCTION}

The coffee powder has been used for hundreds of years to treat wounds and used by people without raising doubts because of its effectiveness in wound healing. Indonesian general physicians who worked in the periphery reported the efficacy of homemade coffee for wound treatment. At present, the management of wounds might cause discomfort due to repeated debridement, frequent dressing replacement, and higher cost. The previous studies showed the coffee powder could heal faster than gauze-saline, topical antibacterial (Ag-sulfadiazine), foam pad, and hydrocolloid pad [1]. An investigation at the time of a wound dressing replacement has also shown the epithelial cells adhered and clung to the wound dressing [2]. It indicates there is a kind of trauma by wound dressing replacement. Although the injury can heal by repopulated cells, it affects a longer wound healing time.

\section{THE COFFEE'S ANTIOXIDANT, ANTI-INFLAMMATORY AND ANTIBACTERIAL}

With proper and closed storage, no microbes can grow on the coffee grounds. Thus, the coffee can be ready to use as a costless wound dressing. The caffeinated coffee powder (the whole coffee powder) is applied for the wound healing process because it is more reasonable to use it than caffeine alone. Literature provides information regarding in vitro caffeine additions that do not support skin tissue's epithelial proliferation. The author's clinical investigation since 2003, it was more than 300 wound cases using coffee to treat acute and chronic wounds have shown its sophistication [1]. It provides antioxidant, anti-inflammatory, antibacterial, activated skin microcirculation, and sweet scent [1]. Antibactericidal mechanism of coffee mixed with wound fluid produced hydrogen peroxide and hyperosmotic juice kill the bacterial cells [3]. The coffee powder has created a better wound healing than the foam pad and the gauze-saline, and it causes less detachment of the wound surface cells [2]. It is known as a topical wound cover that accelerates the growth of new cells in therapeutic performance. The coffee powder can manage through all therapeutic injury stages, and shorten the inflammation, provide collagen proliferation, and better epithelialization to quicker healing [1]. It is almost available everywhere and straightforward to apply. The powder will readily dissolve in wound fluid, and it starts with its positive interaction in wound scab and against the oxidative components, bacterial existence, and inflammatory process. During the phase of hemostasis, the coffee granule will provoke platelet adhesion and terminate any bleeding. Its benzopyrone capacity will be advantageous to stimulate macrophage activities against foreign biological matter [4]. It will excite proangiogenic growth hormones, such as VEGF (Vascular Endothelial Growth Factor), TGF (Transforming Growth Factor), FGF (Fibroblast Growth Factor), PDGF (Platelet-Derived Growth Factor). It is an essential contribution of coffee, particularly in the second phase 
(inflammation). Coffee can eliminate or inhibit the prolongation of the acute inflammatory phase. The shortness of the inflammatory phase causes wound healing to go into the macrophage activity's proliferation phase. Naturally, wounds (primary or secondary intention healing) in humans can close (cure) with sufficient endurance, without drug administration [5]. It occurs in healthy animals. The wound will have difficulty recovering in people with diabetes mellitus with high sugar levels. The wound can heal faster with ground coffee, honey, turmeric, or aloe vera, or other medicinal plants in literature. Thus, all phases of healing are quickly passed and swiftly resolved. Natural wound healing does not require trauma (micro deformation or macro deformation) to stimulate healing [6]. It does not need expensive synthetic wound covers or specialized operations courses. Wound closure should be simple, efficient to heal, easy to practice, and lower cost. In a healthy situation, immediately after a wound occurs, the tissue cells directly work to stop light bleeding, eradicate bacterial contaminants, against inflammation, and overcome oxidants. Then start with capillary cell proliferation (angiogenesis). Epithelialization starts from the wound's edge to the center of the damage. The last is to do remodeling, which reduces the excess and adds to collagenous scar tissue shortcomings.

\section{The EQUILIBRIUM OF CAFFEINATED-COFFEE}

Caffeinated coffee has an equilibrium ability with the best wound healing capability [1]. Many clinical non-ischemic cases with blood-controlled glucose diabetes or non-diabetes, postoperative wounds with secondary intention healing, or primary intention healing managed to recover using coffee powder [1]. The results were fulfilled and without any complications. Caffeine is an adenosine-receptor inhibitor that impairs epithelialization and keratinocyte proliferation [7]. The molecule of the coffee bean is always in equilibrium by nature. Coffee is helpful during wound healing, mainly based on its capabilities [1]. The coffee ground can be a hemostatic agent to stop capillary bleeding. Coffee supports to halt inflammation in the acute wound and prevents chronicity. Coffee anticipates the formation of excessive scar (hypertrophic scar, keloid) in secondary intention wound healing.

\section{The New PARAdigm OF Wound MANAgEMENT}

The coffee creates the decreased MMP-1 level in wounds infected by Staphylococcus aureus ATCC 25923. It was the fastest wound healing compared with hydrocolloids (statistically not significant) or gauze-saline (statistically significant) in the rat [8]. Repeated unnecessary debridement should not be done because harmful and traumatic to the wound tissue, except for carefully removing dead tissue. The repeated washing with saline or distilled water will elevate the wound tissue's $\mathrm{pH}$ and cause bacterial contamination, which allows a new inflammation [9]. The bacteria could enter the wound from the hair follicles of the surrounding healthy skin while washing. [10].

The ideal topical wound dressing should have the significant ability of antioxidant, anti-inflammatory, antibacterial, and pleasant scent. It also has the capabilities of economical and stimulates skin microcirculation [1]. The coffee powder has all those capabilities. The wound can cure using coffee powder with a straightforward and efficient procedure, with no infectious complications in our series [11]. During every dressing replacement, the superficial cells clung to the dressing [11]. Thus, frequent dressing replacement of an adherent dressing, especially by sticky gauze-saline, causes trauma to the cells in the wound surface and may slow down the healing. The superficial cells will not cling to the dressing because a thin layer of coffee powder deliberately remained in the wound. The remained layer of coffee powder (Fig. 1) prevents trauma to the wound's new growth cells during replacement. The remaining coffee powder was safe because it is an antioxidant, antiinflammatory, and antibacterial. Repeated debridement should perform if necessary because traumatic and harmful to the new growing cells. There will always be a release of the cells, except using coffee powder. The frequent and repeated washing elevates the wound's $\mathrm{pH}$ by wetting and diluting the wound area and injuring the growing cells [10]. The hair follicle's bacteria will quickly enter the wound part and produce bacterial contamination. Coffee has proved an efficient wound dressing and accelerates wound healing since less traumatic and offers superior wound handling [11], [12]. A coffee layer is essential to prevent the wound surface (Fig. 1) from any trauma to the new growing cells [13]. The procedure explained above is a new one called the New Paradigm of Wound Management. It is an effective method for acute and chronic wounds (Fig. 2). It is low cost, easy to get, acceptable scar, non-traumatic, and not scary to the patients. A diabetic wound patient, which caused her mobilization using a wheelchair for almost two years. The damage was severe enough to the soft tissue near the calcaneus bone, but no sign of osteomyelitis or osteoporosis with the topically treated coffee powder. During the healing process using coffee powder, she refused any invasive procedure, including debridement! It resulted in a nice acceptable scar in six months. A week after the wound closed, she can walk again using an assisted walker. During the treatment process, she followed a plant-based lifestyle and mild exercise. Its powder mixed with the wound fluid. Coffee proteinases endorsed the autolysis procedure, which supported the wound healing phases [9]. The coffee powder will absorb the infective wound fluid and decline productive exudate. Coffee's antimicrobial capacity actively supports contaminated microbial eradication effectively. The coffee has benzopyrone. It is a defense mechanism stimulating the macrophages to phagocytize the microbes, foreign bodies, debris and clean the wound [4]. The wound cover with coffee powder functions against bacterial contamination and plays an active role in the healing process. It reduced the inflammatory cytokines (TNF- $\alpha$, IL-1, IL-6, IL-12) production [14].

Research shows the effectiveness of coffee grounds on secondary intention healing wounds measuring $5 \mathrm{~cm} \times 5 \mathrm{~cm}$, $0.5 \mathrm{~cm}$ deep requires 80 grams of coffee powder. An extensive injury needs coffee grounds until the healing phase reaches granulation tissue growth, then skin grafting can be continued.

Wound healing in patients with type 2 diabetes mellitus 
accompanied by regulation of blood sugar. In patients without blood sugar regulation, coffee grounds were not successful in healing wounds.

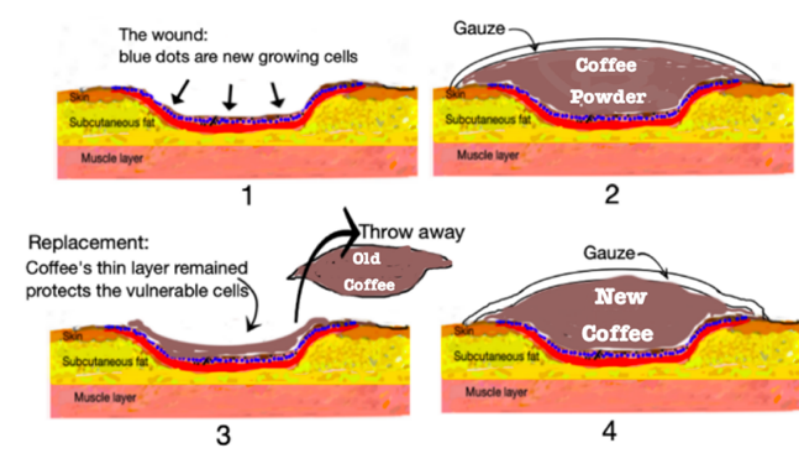

Fig. 1. Coffee for wound healing.

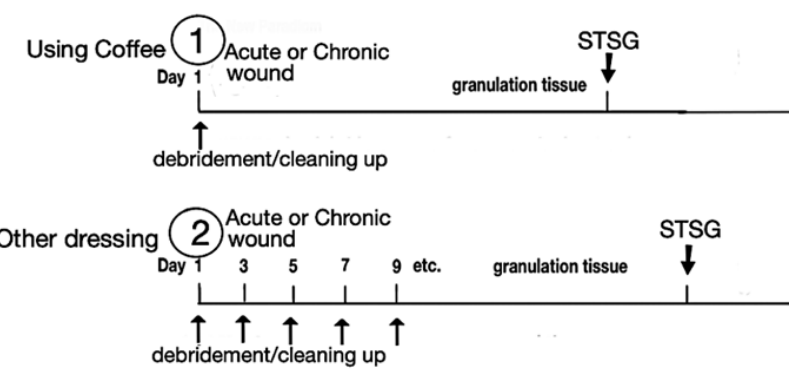

Fig. 2. 1) Using coffee for wounds. 2) Using other dressing.

\section{ECONOMIC IMPACT}

Coffee for wounds has the advantage of making wound management lower economical and more straightforward in the procedure of injury topical replacement. Today wound management applying Modern Wound Dressing, Negative Pressure Wound Treatment (NPWT), Hyperbaric Oxygen tank, is with higher costs, is a challenge. Probably coffee is the best answer, as much more reasonable and economic comparison to them. It is available in the periphery, far from any health provider facilities. For minor injuries that need a simple procedure, coffee is much simple care for everyone. The new paradigm in wound management using coffee powder can help solve wound problems. Its simplicity in wound management procedures helps improve public health efforts.

\section{EfFective HeAling AND ACCEPtable SCAR}

Coffee grounds are an effective wound healing with acceptable scar tissue [1], [12], [13]. Minimum trauma to the wound tissue is consequently applied. It suppresses the emergence of bacterial contamination and is an antioxidant, anti-inflammatory, and antibacterial, very efficient scars. The coffee extract has the performance to stimulate the epidermal stem cells of the normal skin and has an essential compound of 6-furfuryladenine (kinetin) that has a robust capacity in cell regeneration [15]. minimum detached wound cells compared to other dressings. The utilization of coffee's antioxidant, anti-inflammatory, and antibacterial capacities anticipates the injury of the new growth of epithelial cells at the wound bed, better cell proliferation, proper scar formation, pleasant scent, costeffectiveness, and scary to the patients. Its simplicity in wound management procedures helps improve public health efforts. Hence, it suggested that the coffee powder has the capabilities of the best topical wound dressing.

\section{ACKNOWLEDGMENT}

None.

\section{REFERENCES}

[1] Yuwono HS. The New Paradigm of Wound Management Using Coffee Powder. Global Journal of Surgery. 2014; 2(2): 25-29.

[2] Yuwono HS, Nugroho BE. Epithelial cells detachment by wound dressing replacement will slow wound healing. European Wound Management Association (EWMA) Conference 2017, AmsterdamRAI, Netherlands, May 3-5, 2017.

[3] Mueller U, Sauer T, Weigel I, Pichner R, Pischetsrieder M. Identification of $\mathrm{H} 2 \mathrm{O} 2$ as a major antimicrobial component in coffee. Food \& Function. 2011; 2(5): 265-272.

[4] Mayer LSL, Stoll DA, Geisen R, Schmidt-Heydt M. Benzopyrone Coumarin Leads to an Inhibition of Ochratoxin Biosynthesis in Representatives of Aspergillus and Penicillium spp. via a Type of Feedback Response Mechanism. Journal of Food Protection. 2014; 77(4): 647-652.

[5] Moon CH, Crabtree TG. New Wound Dressing Techniques to Accelerate Healing. Current Treatment Options in Infectious Diseases. 2002; 5: 251-260.

[6] Herliani T, Yusyahadi FA, Yuwono HS, Noor IM, Sakinah K, Djajakusumah T. The experimental wound healing using coffee powder and honey compared to NPWT. International Journal of Medicine and Pharmaceutical Science. 2018; 8(6): 31-38.

[7] Ojeh N, Stojadinovic O, Pastar I, Sawaya A, Yin N, Tomic-Canic M. The effects of caffeine on wound healing International Wound Journal. 2016; 13(5): 605-613.

[8] Vicente SJV, Queiroz YS, Gotlieb SLD, da Silva Torres EAF. Stability of Phenolic Compounds and Antioxidant Capacity of Regular and Decaffeinated Coffees. Brazilian Archives of Biology and Technology. 2014; 57(1): 110-118.

[9] Winata AA, Yuwono HS, Hapsari P. Correlation between MMP-1 Responses and Epithelialization of Acute Staphylococcus aureus Infected Wounds Treated by Coffee powder, Saline gauze, and Hydrocolloid. Interational Journal of General Medicine and Pharmacy. 2017; 6(5): 31-38.

[10] Arimbi D, Yuwono HS. pH of Wound Fluids Treated Using Coffee Powder and Bacitracin-Neomicin Powder. Global Journal of Surgery. 2016; 4(1): 9-11.

[11] Yuwono HS. The New Paradigm of Wound Management: A Must to Follow. Hospice and Palliative Medicine International Journal. 2017; 1(4): 97-99.

[12] Yuwono HS. A Case of Diabetic Wound: The Coffee Powder Protects the Growth of Cells on the Wound Bed. American Journal of Medical Case Reports. 2019; 7(5): 94-96.

[13] Garna H, Yuwono HS, Tejasari M. Vulnerable Wound-base Cells Protected by Coffee Powder to Better Healing. American Journal of Medical Case Reports. 2019; 7(9): 214-216.

[14] Ho KV, Schreiber KL, Park J, Vo PH, Lei Z, Sumner LW, et al Identification and Quantification of Bioactive Molecules Inhibiting Pro-Inflammatory Cytokine Production in Spent Coffee Grounds Using Metabolomics Analyses. Frontiers in Pharmacology. 2020; 11(229): $1-11$.

[15] Miastkowska M, Sikora E. Anti-Aging Properties of Plant Stem CellExtracts. Cosmetics. 2018; 5(4): 1-8.

\section{CONCLUSION}

The remained thin layer of coffee powder has created 


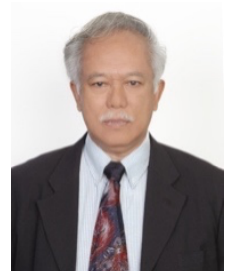

H. S. Yuwono was born in Kuningan, West Java, Indonesia, January 26, 1947. Surgeon, University Padjadjaran, Bandung, Indonesia. PhD degree University Amsterdam, The Netherlands 1988; vascular surgery in Leiden Academic Hospital, The Netherlands 1990. Major field of study: wound healing using coffee powder.

His previous job Professor of Surgery Univ. Padjadjaran until 2017, Univ. Islam Bandung started 2017 (current job) Jalan Tamansari 22, Bandung, Indonesia. Published books: 1) A textbook of Vascular Surgery in Bahasa Indonesian [Vascular Surgery. Science and practical experiences], Bandung, Indonesia: PT. Refika Aditama. 2) Coffee. The New Paradigm of Wound Management, Bandung, Indonesia: PT. Refika Aditama. Published articles: 1) The New Paradigm of Wound Management using Coffee Powder. Invited Lecture in Dubai, The 3rd Biotechnology World Conference, February 10-12, 2014. 2) Coffee for the Management of Diabetic Foot Wounds. A New Paradigm. Abstract code: P18.07, The 7th International Symposium on the Diabetic Foot, Den Haag, The Netherlands. May 21-23, 2015. 3) "Epithelial Cells Detachment by Wound Dressing Replacement Will Slow Wound Healing". E-Poster presentation in European Wound Management Association Conference, Amsterdam-RAI, The Netherlands, May 3-5, 2017. Current and previous research interest: Coffee for wound healing.

Prof. Yuwono is a member of Indonesia Surgeon Association, Indonesia Medical Doctor Association, Indonesia Vascular Surgeon and Endovascular (PESBEVI), Asian Vascular Society, The Indonesian Society for Microcirculation and Phlebology, 1997 (Founder), Professor' council of Univ. Padjadjaran and UNISBA. Appreciation from the Rector Magnificus Univ. Padjadjaran: "Coffee for wound healing" May 25, 2012. Coffee Book Award winner from the Republic Indonesia National Library, Jakarta November 17, 2020. Two Patents awarded in 2020 about wound's treatment using coffee powder. 